obliged to adopt an assumption for which there is no proof, that the transient responses generated in the two classes of receptor will exactly cancel out in all the putative 'luminance' channels; we must suppose that the weightings of inputs from the different receptors are in a constant ratio for all channels and that the transient waveforms of different signs are symmetrical. Transients in space and time are biologically significant and it would be odd if the retina allowed them to be lost in this way.

A second pitfall may lie in relating the detection measurements mentioned above directly to the classical phenomenological measurements (see for example Hurvich \& Jameson Psychol. Rev. 64, 384; 1957) that kept alive the concept of opponent processes during a period of neglect. General understanding of the first stage of colour vision was earlier held back by a widespread but unjustified belief that the peak sensitivities of the three receptors should correspond to phenomenologically primary colours, red, green and blue; it may equally be a mistake to suppose now that the opponent processes of the retina must be directly related to the phenomenological oppositions of blue and yellow and of red and green. S. Zeki (University College, London) presented his measurements of the spectral sensitivities of individual nerve cells in Area V4 of the cerebral cortex of the rhesus monkey; even at this central level, although many cells have narrow bandwidths $(10-20 \mathrm{~nm})$, the peak sensitivities do not cluster very tightly at the four phenomenologically pure hues, blue, yellow, green and red, but are distributed throughout the spectrum, with possibly a gap at $560-570 \mathrm{~nm}$.

\section{Colour constancy}

As we view an object from different distances and angles or under different illuminations, our perception changes much less than does our retinal image. This stability of our perception is known as perceptual constancy. It is an everyday observation that a sheet of white paper looks equally white in daylight and in the yellowish illumination of domestic tungsten light, although if outdoor film were used to photograph the paper indoors, the resulting transparency would have a strong yellow cast. The correction achieved by our visual system (but not by the photographic process) is called colour constancy. One of the most interesting results presented at the meeting was Zeki's finding that some individual cortical cells show colour constancy: if an array consisting of many different coloured patches is placed before a monkey, and, say, a green patch is arranged to cover the receptive field of a green-sensitive cell, the cell responds to the patch despite gross changes in the spectral composition of the illumination falling on the array; but the cell does not respond if, say, a red patch is placed in its receptive field and the overall illumination is adjusted so that the spectral composition of the flux reflected from the red patch was identical to the flux previously reflected from a green patch to which it did respond (Zeki Nature in the press). Under the latter condition, of course, the patch looks red, not green, to a human observer (see Land Sci. Amer. 237, December; 1977) just as, in the classical demonstration of size constancy scaling, an after-image of fixed retinal dimensions looks of different sizes when projected on surfaces at different distances.

\section{Colour preferences}

The layman reasonably expects the colour scientist to be much concerned with people's colour preferences, but the experimental study of colour preference has an unhappy history of inconsistent results and has been neglected in recent years. Reviewing this field for the meeting, I.C. McManus (Bedford College and St. Mary's Hospital, London) noted that earlier

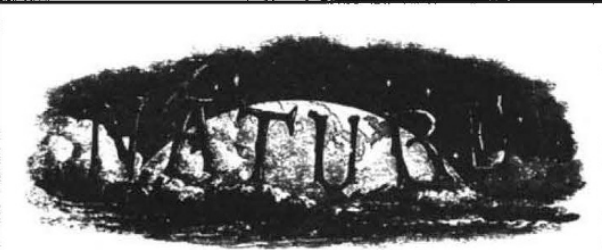

100 years ago

\section{THE CRAYFISH}

Common and lowly as most may think the crayfish, it is yet so full of wonders that the greatest naturalist may be puzzled to give a clear account of it." These words from von Rosenhof, who in 1755 contributed his share to our knowledge of the animal in question, are cited by Prof. Huxley in the preface to the careful account of the English crayfish

1 "The Crayfish: an Introduction to the Study of Zoology." By T.H. Huxley, F.R.S. (London: Kegan Paul, 1880.) studies had confounded variation in hue with variations in saturation and lightness, and he described a study, performed in Cambridge in collaboration with A. Jones and J. Cottrell, in which this failure was corrected. Another feature of this new study was the use of the 'method of paired comparisons': each of a large set of coloured chips was paired with every other chip and the subject was asked to express a preference between the members of each possible pair, thus allowing the experimenter to derive a measure of internal consistency. When saturation and lightness are held constant, the population shows a clear preference for blue and a clear dislike of yellow. Further analysis shows differences between individuals but consistency within an individual's data; differences between individuals seem to be almost entirely due to differences in lightness preference and saturation preference, with very small inter-subject differences in hue preference. and its immediate congeners, which forms the latest volume of the International Scientific Series. The book is not designed for "general readers," those somewhat luxurious but presumably intelligent persons for whom so much scientific knowledge is chopped and spiced at the present day. It is, as we gather from the author's statement, intended as an introduction to serious zoological study, for those who will turn over its pages, crayfish in hand, and carefully verify its statements as to details of structure with scalpel and microscope. To these and also to those who are already well versed in crustacean anatomy, the book will have great value and interest; to the latter more especially, as showing how in the careful study of one organism we are "brought face to face with all the great zoological questions which excite so lively an interest at the present day," and as an exhibition of that "method by which alone we can hope to attain to satisfactory answers of these questions."
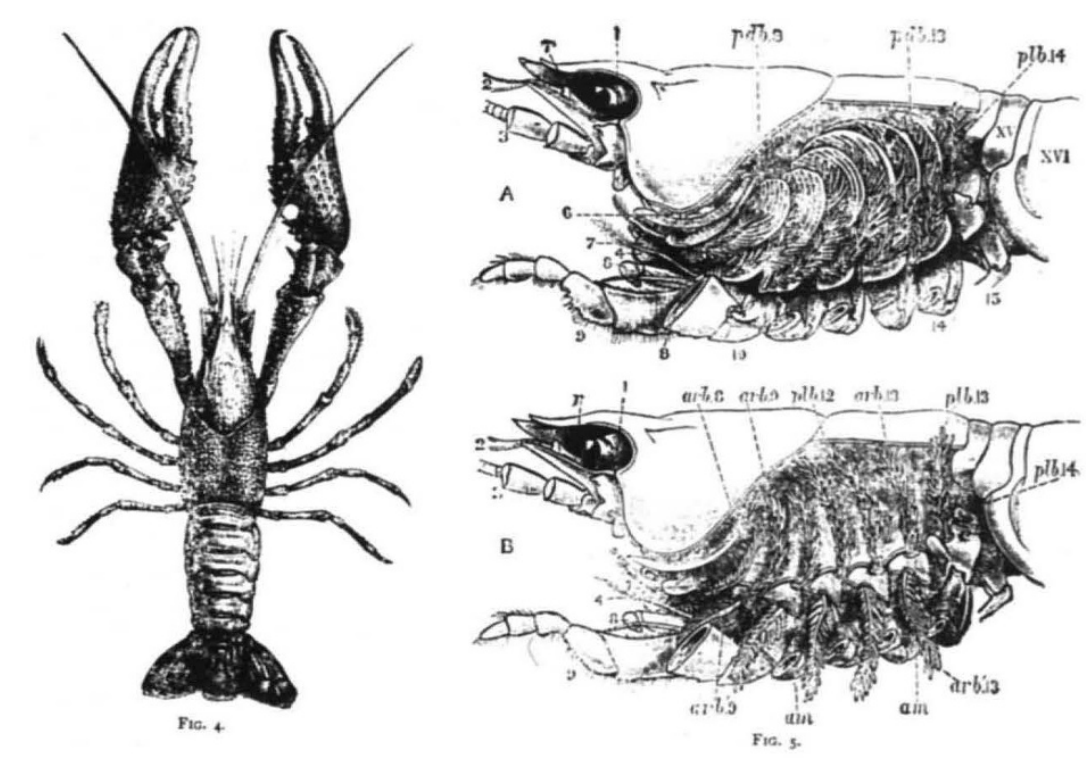

From Nature 21, 12 Feb., 353; 1880. 